\title{
Reentrant vortex lattice transformation in four-fold symmetric superconductors
}

\author{
N. Nakai, P. Miranović, M. Ichioka and K. Machida \\ Department of Physics, Okayama University, 700-8530 Okayama, Japan
}

(Dated: October 22, 2018)

\begin{abstract}
The physics behind the rhombic $\rightarrow$ square $\rightarrow$ rhombic flux line lattice transformation in increasing fields is clarified on the basis of Eilenberger theory. We demonstrate that this reentrance observed in $\mathrm{LuNi}_{2} \mathrm{~B}_{2} \mathrm{C}$ is due to intrinsic competition between superconducting gap and Fermi surface anisotropies. The calculations reproduce not only it but also predict yet not found lock-in transition to a square lattice with different orientation in higher field. In view of physical origin given, this sequence of transitions is rather generic to occur in four-fold symmetric superconductors.

PACS numbers: 74.60.Ec, 74.60.-w, 74.70.Dd
\end{abstract}

The morphology of equilibrium flux line lattice (FLL) in type-II superconductors, its symmetry and orientation relative to the crystallographic axes, is determined by microscopic electronic properties, particularly by Fermi surface topology and superconducting pair symmetry. In general, the shape and orientation of FLL cell will change with field direction trying to adjust the electronic anisotropy of underlying crystal [1]. In spite of a long research history which dates back to the pioneering small angle neutron scattering experiment by Cribier et al. [2] on $\mathrm{Nb}$, problems associated with FLL are still lively discussed even on seemingly simplest elemental metal $\mathrm{Nb}$ [3]. FLL symmetry transformation under varying applied field is one of the topics which attracted much of the attention of vortex physics community recently. The effect became known in the early 70's when the low field rhombic-to-square FLL transition has been observed first in $\mathrm{PbTl}$ [1]. Renewed interest in this phenomena came, after similar transformations has been detected in a number of superconductors: $(\mathrm{Re}) \mathrm{Ni}_{2} \mathrm{~B}_{2} \mathrm{C}(\mathrm{Re}=\mathrm{Lu}, \mathrm{Y}, \mathrm{Er}, \mathrm{Tm})$ [5, 6, 7], $\mathrm{V}_{3} \mathrm{Si}$ [8] and high- $T_{c}$ cuprate $\mathrm{La}_{1.83} \mathrm{Sr}_{0.17} \mathrm{CuO}_{4}$ [9. All of them appear to be four-fold symmetric crystals: cubic or tetragonal.

Recently, Eskildsen et al. [10 discovered a remarkable reentrant transition of the rhombic FLL symmetry for $\boldsymbol{H} \| \boldsymbol{c}$ in $\mathrm{LuNi}_{2} \mathrm{~B}_{2} \mathrm{C}\left(H_{c 2,0} \sim 9 \mathrm{~T}\right)$. Upon increasing $H$, the rhombic lattice changes into square one and then backs again at a higher field. With increasing temperature the rhombus-square boundary bends away and never crosses $H_{c 2}$ line. Combining its result with other experiments such as STM [6, 11], $\mu$ SR [12 and Bitter decoration 13], square FLL region is confined in a small area just above $H_{c 1}$ and up to $\sim 2-3 \mathrm{~T}$ on $(H, T)$ plane. High field square-to-rhombus transition is detected in $\mathrm{TmNi}_{2} \mathrm{~B}_{2} \mathrm{C}$ [7] as well, though this is a magnetic member of borocarbide family. It is quite interesting to remember $\mathrm{Nb}$ case. The FLL in this cubic system for $\boldsymbol{H} \|(001)$ exhibits square-to-rhombic transition as $T$ increases [14. Although the definite phase diagram is not established yet, temperature dependence of FLL symmetry alone suggests an isolated region of stable square lattice in $H$ vs. $T$.
It was realized early 15 that a certain four-fold anisotropy in plane perpendicular to the applied field, such as the Fermi velocity $v_{F}$, can drive low field rhombus-to-square transformation. In the in-plane anisotropy modeled by $v_{F}(\theta)=v_{F}\left(\frac{\pi}{8}\right)(1+\beta \cos 4 \theta)(\theta$ polar angle), square FLL $\square_{v}$ with nearest neighbors oriented along the velocity minimum will be stabilized in low fields if anisotropy degree $\beta$ is large enough. The same is true for the four-fold gap anisotropy alone $|\Delta(\boldsymbol{r}, \theta)|^{2}=$ $\left|\Delta\left(\boldsymbol{r}, \frac{\pi}{8}\right)\right|^{2}(1-\alpha \cos 4 \theta)$, when square FLL $\square_{g}$ (nearest neighbors along gap minimum) tends to be stabilized. These are schematically shown in Fig.1. The nontrivial question is why square FLL is unstable at high fields and what the actual phase diagram looks like? Contrary to the answer given by Gurevich and Kogan [16] who consider it due to thermal fluctuation near $H_{c 2}$, here we investigate two indispensable anisotropic effects on the same footing. The interplay of gap and Fermi surface anisotropy indeed can give rise to the reentrant FLL transition and further square lock-in transition in a higher field.

There is firm evidence in $\mathrm{LuNi}_{2} \mathrm{~B}_{2} \mathrm{C}$ and $\mathrm{YNi}_{2} \mathrm{~B}_{2} \mathrm{C}$, which we regard as essentially the same systems, to show the existence of both anisotropies. As for the gap anisotropy, various bulk measurements, including the $H$ dependent linear specific heat coefficient $\gamma(H)$ (Volovik effect) 17], the thermal conductivity 18, and angleintegrate photo-emission [19], all show a substantial gap anisotropy. More recently the angle-resolved thermal conductivity [20] under $H$ exhibits that the gap is vanishingly smaller in (100) than in (110). This is consistent with the spatial extension of the zero-bias peak observed by STM [21]. As for the Fermi velocity anisotropy, when interpreted through simple four-fold harmonic variation, band calculation [22] told us that the Fermi velocity is larger in (100) than in (110), or the angle resolved density of states (DOS) $N(\theta) \sim 1 / v_{F}(\theta)$ is smaller along (100) compared to (110). Note that the sense of two anisotropies mentioned is what we would naively expected because the larger gap $\Delta(\theta=0)$ should develop in larger $N(\theta=0)$. The built-in tendency to stabilize two different orientation $\square_{v}$ and $\square_{g}$ of square lattice ul- 

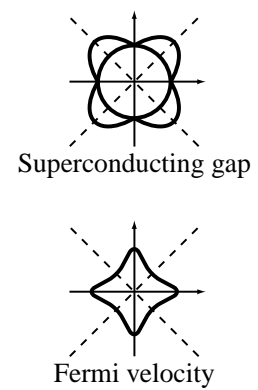
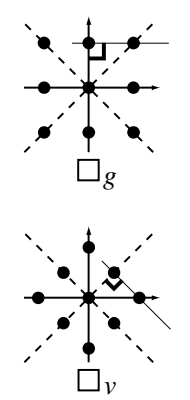

(010)

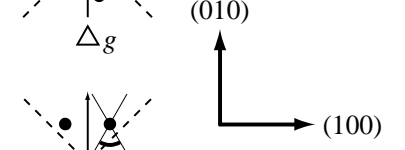

FIG. 1: Rhombus and square FLL cell orientations relative to the crystal. The vortex center is shown by $\bullet$. $\alpha>0, \beta>0$.

timately leads to the rich vortex phase diagram, including the reentrant as we will see shortly.

Apart from the limiting cases: London model at $H \ll$ $H_{c 2}(T)$ and Ginzburg-Landau (GL) model at $T \approx T_{c}$, there is no handy and convenient approximate scheme to describe microscopic vortex properties deep in $(H, T)$ plane. Therefore we resort to quasi-classical Eilenberger equations [23] valid for $k_{F} \xi_{0} \gg 1\left(k_{F}\right.$ : the Fermi wave number and $\xi_{0}$ : the coherence length) a condition met in most of superconductors. Eilenberger equations read as $(\hbar=1)$

$$
\begin{gathered}
\left(2 \omega+\boldsymbol{v}_{F}(\theta) \cdot \boldsymbol{\Pi}\right) f(\omega, \boldsymbol{r}, \theta)=2 \Delta(\boldsymbol{r}, \theta) g(\omega, \boldsymbol{r}, \theta), \\
\left(2 \omega-\boldsymbol{v}_{F}(\theta) \cdot \boldsymbol{\Pi}^{*}\right) f^{\dagger}(\omega, \boldsymbol{r}, \theta)=2 \Delta^{*}(\boldsymbol{r}, \theta) g(\omega, \boldsymbol{r}, \theta) .
\end{gathered}
$$

Here $\boldsymbol{\Pi}=\boldsymbol{\nabla}+\left(2 \pi i / \Phi_{0}\right) \boldsymbol{A}$ is gauge invariant gradient, $\boldsymbol{A}$ is vector-potential and $\Phi_{0}$ is flux quantum. $\omega=\pi T(2 n+1)$ with integer $n$ is Matsubara frequency. Normalization condition for Green's function $g^{2}+f f^{\dagger}=1$. The pairing interaction is assumed separable $V\left(\theta, \theta^{\prime}\right)=\bar{V} \phi(\theta) \phi\left(\theta^{\prime}\right)$ so that gap function is $\Delta(\boldsymbol{r}, \theta)=\Psi(\boldsymbol{r}) \phi(\theta)$. We consider two-dimensional case with cylindrical Fermi surface. Four-fold models for Fermi velocity $v_{F}(\theta)=\bar{v}_{F}(1+\beta \cos 4 \theta) / \sqrt{1-\beta^{2}}$ and gap anisotropy $\phi(\theta)^{2}=\phi\left(\frac{\pi}{8}\right)^{2}(1-\alpha \cos 4 \theta)$ have been adopted. Here $\theta$ is polar angle relative to (100) axis. Constant $\phi\left(\frac{\pi}{8}\right)^{2}=1 /\left(1+\left(1-\sqrt{1-\beta^{2}}\right) \alpha / \beta\right)$ is chosen to assure the same $T_{c}$ and DOS $N_{0}$ for any value of anisotropy parameters $\alpha$ and $\beta$. We are most interested in case when $\alpha$ and $\beta$ are of the same sign. Then positions of gap and velocity minima are $45^{\circ}$ rotated to each other (see Fig.1) and we have a competing effect. The self-consistent equations for the gap function $\Psi(\boldsymbol{r})$ and vector-potential $\boldsymbol{A}$ are

$$
\begin{aligned}
& \Psi(\boldsymbol{r}) \ln \frac{T_{c}}{T}=2 \pi T \sum_{\omega>0}\left[\frac{\Psi(\boldsymbol{r})}{\omega}-\left\langle\frac{\phi(\theta) f}{v(\theta)}\right\rangle\right], \\
& \boldsymbol{\nabla} \times \boldsymbol{\nabla} \times \boldsymbol{A}=-\frac{16 \pi^{3}}{\Phi_{0}} N_{0} T \bar{v}_{F} \sum_{\omega>0} \operatorname{Im}\langle g \boldsymbol{u}\rangle .
\end{aligned}
$$
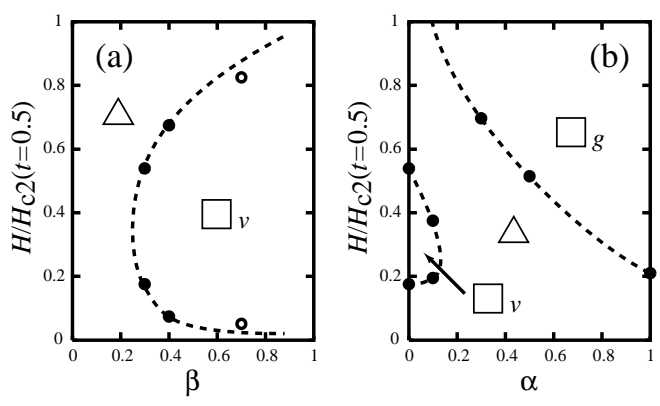

FIG. 2: FLL phase diagram at $T / T_{c}=0.5$. a) $\beta$-dependence $(\alpha=0)$, o shows where $\square_{v}$ is stable. b) $\alpha$-dependence $(\beta=$ 0.3). Broken lines are guide for the eye.

Here, $v(\theta)=v_{F}(\theta) / \bar{v}_{F}$ and $\boldsymbol{u}=(\cos \theta, \sin \theta)$ is unit vector along the Fermi velocity $\boldsymbol{v}_{F}=v_{F}(\theta) \boldsymbol{u}$. For average over Fermi surface, $\langle\ldots\rangle=(1 / 2 \pi) \int \ldots d \theta$. Extra factor $1 / v(\theta)$ in averages came from angle resolved DOS $N(\theta)=N_{0} / v(\theta)$ on Fermi surface.

We have performed extensive numerical computations by the so-called explosion method (see Ref. 24 for details) for various values of anisotropy parameters $\alpha$ and $\beta$ in a high GL parameter case $\kappa=100$. The self-consistent solution yields a complete set of the physical quantities: the spatial profiles of the order parameter $\Psi(\boldsymbol{r})$ and the magnetic field $H(\boldsymbol{r})$, and the local density of states around a vortex core. The free energy density is given by

$F=\frac{\overline{H^{2}(\boldsymbol{r})}}{8 \pi}-2 \pi T N_{0} \sum_{\omega>0}\left\langle\overline{\frac{1-g}{1+g} \frac{\phi(\theta)\left(\Psi^{*} f+\Psi f^{\dagger}\right)}{2 v(\theta)}}\right\rangle$

Here, $\bar{a}=\left(B / \Phi_{0}\right) \int_{\text {cell }} a d \boldsymbol{r}$. Free energy should be minimized with respect to the FLL symmetry and its orientation relative to the crystallographic axes. Numerics is backed up by analytical calculations. Namely we also solve these analytically at the two limiting cases; (1) near $H_{c 2}(T)$ and $(2) H \ll H_{c 2}(T)$ to gain physical insights. For analytical results we considered FLL cell shaped as rhombus with apex angle in interval $\left[60^{\circ}, 90^{\circ}\right]$. Two different orientations of rhombus cell are compared: rhombus diagonals along gap minimum (velocity maximum) and along velocity minimum (gap maximum) (see Fig. 1). Since the numerical computation is very demanding and time consuming, we limit ourselves to the four configurations (apex angles $60^{\circ}$ and $90^{\circ}$ for each of two orientations) as shown in Fig.1.

In Fig. 2(a) we show the phase diagram for $t=T / T_{c}=$ 0.5 and $\alpha=0$, where the square lattice $\square_{v}$ becomes stable if the Fermi velocity anisotropy $\beta$ exceeds a certain value. It shows also that the triangular lattice is always stabilized at lower and higher $H$. Thus even without the gap anisotropy $(\alpha=0)$ the reentrant transformation can be induced. This can be understood by looking at the self-consistency Eq. (3). It is seen that factor $1 / v(\theta)$ plays the role of gap anisotropy even in isotropic pairing 


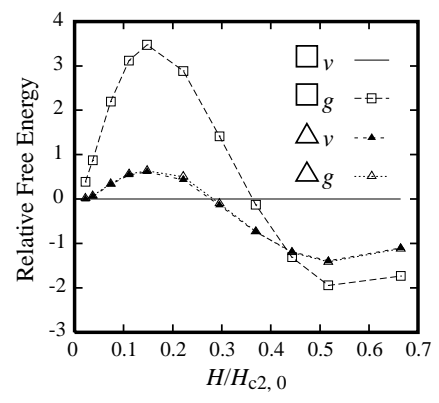

FIG. 3: Field dependence of free energy for $\triangle_{v}, \triangle_{g}$ and $\square_{g}$ relative to $\square_{v}$. $T / T_{c}=0.25, \alpha=\beta=0.3$. Free energies of $\triangle_{v}$ and $\triangle_{g}$ are almost identical.

case $(\phi(\theta)=1)$.

The gap anisotropy further induces a rich variety of the phase diagram. In Fig. 2(b) we show it when the moderate velocity anisotropy $\beta=0.3$ is taken. We see that at the large $\alpha$ cases the triangular lattice directly changes into the square lattice $\square_{g}$. This is a similar situation to the $d$-wave case with the isotropic Fermi velocity [24]. The preferred orientation perfectly coincides with the nodal direction, namely, nearest neighbors of FLL are along (100) (along (110)) in $d_{x y}\left(d_{x^{2}-y^{2}}\right)$ pairing. As $\alpha$ decreases, this $\square_{g}$ lattice region shrinks. At lower fields yet another transformation from the triangular to square $\square_{v}$, rotated by $45^{\circ}$ relative to $\square_{g}$, emerges. The most complicated case is at moderate values of $\alpha$ and $\beta$, leading to successive lattice transformation $\triangle \rightarrow \square_{v} \rightarrow \triangle \rightarrow \square_{g}$ as $H$ increases. In a weaker $\alpha$ case the last lock-in transition is absent.

As a typical example we investigate the case $\alpha=\beta=$ 0.3 in details at lower temperature. In Fig. 3 the four free energy curves are shown as a function of $h=H / H_{c 2,0}$ at $t=0.25$. As $h$ increases the above mentioned successive transformations are clearly illustrated as several crossings. Figure 1 displays the resulting phase diagram in $(H, T)$ plane. It is seen from this that (1) the $\square_{v}$ region is confined to lower $H$ and $T$; (2) its boundary bends away from $H_{c 2}$; (3) the high field region is occupied by the $\square_{g}$ lattice; (4) along $H_{c 2}$ line this terminates at $t=0.56$, below which the rhombic lattice becomes stable.

Let us discuss the physical origin of this intricate phase diagram in connection with the observation in borocarbides. Basically, in order to induce narrowly limited $\square_{v}$ region at low $H$ and $T$ we need the competing effects, each coming from the gap and velocity anisotropies. The gap anisotropy with $\alpha>0$ prefers the lattice $\square_{g}$ with nearest neighbors along (100) direction, while the velocity anisotropy with $\beta>0$ tends to favor $45^{\circ}$ rotated square lattice $\square_{v}$. Thus these anisotropies compete each other. The $\alpha \beta<0$ case does not cause such a frustration in FLL.

The interplay of two anisotropies can be understood

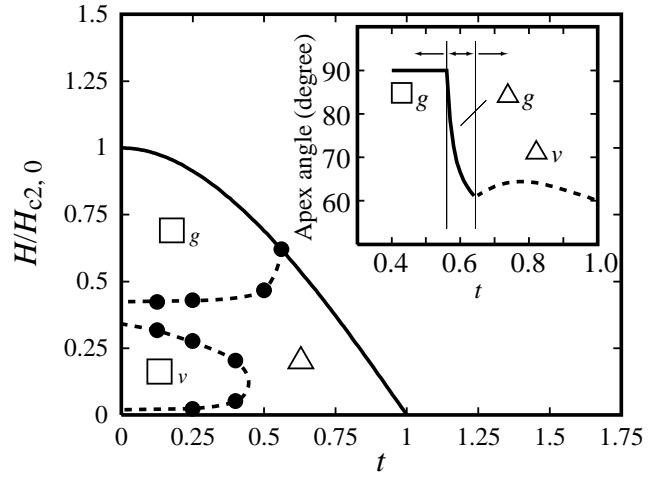

FIG. 4: Phase diagram of FLL for $\alpha=\beta=0.3$. In inset, apex angle variation along $H_{c 2}$ line. The solid (broken) line is for orientation "v" ("g"), see Fig. 1.

by looking at the free energy density at lower $H$ :

$$
F=\frac{B^{2}}{8 \pi} \sum_{q} \frac{1}{1+\lambda^{2} q^{2}+\lambda^{4}\left(t_{x x y y} q^{4}+d q_{x}^{2} q_{y}^{2}\right)}
$$

with $\boldsymbol{q}$ is the reciprocal vector of FLL and

$$
t_{i j l m}=\frac{4 \pi^{4} N_{0} \bar{v}_{F}^{4} T \Psi_{0}^{2}}{\Phi_{0}^{2}} \sum_{\omega>0}\left\langle\frac{\phi^{2}(\theta) v^{3}(\theta) u_{i} u_{j} u_{l} u_{m}}{\left(\omega^{2}+\phi^{2}(\theta) \Psi_{0}\right)^{5 / 2}}\right\rangle,
$$

$\lambda$ is penetration depth, and $\Psi_{0}(T)$ is zero field gap. The extra four-fold anisotropy due to non-local correction appears through the parameter $d=t_{x x x x}+t_{y y y y}-6 t_{x x y y}$ in the above. At lower $T, t_{i j l m}$ contains factor $v^{3}(\theta) / \phi^{3}(\theta)$ which strengthens the tendency towards the velocity anisotropy ( $\beta$ is effectively increased by $\alpha$ when $\alpha \beta>0$ ). Near $T_{c}$ this factor becomes $\phi^{2}(\theta) v^{3}(\theta)$ which weakens the combined anisotropy effect by canceling each other. Therefore the two anisotropies plays different role, depending on $T$, giving rise to the bent transition curve in the $(H, T)$ plane. For a fixed temperature as $H$ increases from $H_{c 1}$, FLL starts with the regular triangle lattice because the electromagnetic interaction between far apart vortices is isotropic, yielding the closed packed hexagonal symmetry just above $H_{c 1}$. As vortices approach each other, proliferating anisotropy in current distribution will squeeze rhombus FLL cell toward square shape. Being dominant at low $T$ region, anisotropy of Fermi velocity will stabilize square lattice $\square_{v}$ as soon as vortices come close to each other with increasing $H$. The observed orientation of $\square_{v}$ with nearest neighbors along (110) is indeed expected in $\mathrm{LuNi}_{2} \mathrm{~B}_{2} \mathrm{C}$ since the band structure calculation indicates that Fermi velocity is larger in (100) than (110) when mapped into our fourfold model [22]. The same result is obtained within nonlocal London model with Fermi velocity anisotropy alone [25]. Upon further increasing $H$, condensation energy gradually takes over the major role in determining the interaction between vortices. Physically it is due to the kinetic energy cost of quasi-particles localized around core 
(see Ref. 24 for details). In high fields, at least in high- $\kappa$ superconductors, the anisotropy in vortex-vortex interaction is exclusively due to the vortex core anisotropy giving rise to the transition from the low field $\square_{v}$ to high field $\square_{g}$ via intermediate rhombic lattice $\left(\square_{v} \rightarrow \triangle \rightarrow \square_{g}\right)$.

Limit of $H \approx H_{c 2}(T)$ allows the analytical solution for free energy

$$
F=\frac{B^{2}}{8 \pi}-\frac{1}{8 \pi} \frac{\left(B-H_{c 2}\right)^{2}}{\mathcal{F}+1}
$$

where

$$
\mathcal{F}=\frac{2 \pi^{2} N_{0} T}{\bar{h}_{s}^{2}} \sum_{\omega>0}\left\langle\overline{\frac{\phi(\theta)}{v(\theta)} f f^{\dagger}\left(\Psi f^{\dagger}+\Psi f\right)}\right\rangle-\frac{\overline{h_{s}^{2}}}{\bar{h}_{s}^{2}} .
$$

Here, $f, f^{\dagger}$ and $\Psi$ are solutions of the linearized Eilenberger equation, $h_{s}$ is magnetic field due to supercurrents, $B=H+\bar{h}_{s}$ is magnetic induction. In the isotropic case $\alpha=\beta=0$ and near $T_{c}$ it reduces to the standard Abrikosov expression $\mathcal{F}=\left(2 \kappa^{2}-1\right) \beta_{A}$. This expression for free energy is valid all along the $H_{c 2}(T)$ line. The result is illustrated in Fig. 4 as the inset showing that the apex angle of the rhombic lattice continuous changes about the regular triangular lattice with $60^{\circ}$. The diagonal of the rhombic lattice rotated by $45^{\circ}$ at $t=0.64$ from (100) to (110), i.e. from $\triangle_{v}$ to $\triangle_{g}$. At a higher field $\triangle_{g}$ lattice is locked in $\square_{g}$. This lock-in point $t=0.56$ is rather nicely connected to the points determined numerically as shown in the main panel in Fig. 4. This implies that our numerical results, which examine the limited number of lattice configurations (4 types), yield a reasonably phase diagram even taking into account general rhombic lattices.

The present calculation does not aim to quantitatively reproduce the actual FLL phase diagram in $\mathrm{LuNi}_{2} \mathrm{~B}_{2} \mathrm{C}$, but to physically understand its possible lattice transformation. Note that so far the observed square lattices in $\mathrm{LuNi}_{2} \mathrm{~B}_{2} \mathrm{C}$ by several methods are all $\square_{v}$, not $\square_{g}$. The $\square_{g}$ phase can occur at further high $H$ if the gap anisotropy is large enough. Since this is the case for $\mathrm{LuNi}_{2} \mathrm{~B}_{2} \mathrm{C}$ as mentioned before, there is a good chance to observe it. According to various thermodynamic and transport experiments the gap anisotropy is extremely large, the recent directional dependent measurements shows the node situated at (100) direction. This unambiguously tells us that $\mathrm{LuNi}_{2} \mathrm{~B}_{2} \mathrm{C}$ belongs to the most interesting competing case $(\alpha>0$ and $\beta>0)$ and also gratifying enough that it belongs to the naively expected case where maximum energy gap is oriented to the maximum DOS direction. So far the investigated field for the vortex structure is limited below $4 \mathrm{~T}$ (compare $H_{c 2,0} \sim 9 \mathrm{~T}$ ).

As for the other materials where the square lattice is found such as $\mathrm{Nb}$ [14], $\mathrm{V}_{3} \mathrm{Si}$ [8] and $\mathrm{Sr}_{2} \mathrm{RuO}_{4}$ [26] we can expect the reentrant transition from the square to rhombic lattice and further lock-in transition to the square lattice with different orientation if the gap anisotropy is strong enough. The recent finding of rhombus-to-square transition at lower $H$ in $\mathrm{La}_{1.83} \mathrm{Sr}_{0.17} \mathrm{CuO}_{4}$ [9] deserves a special attention because in spite of $d_{x^{2}-y^{2}}$ symmetry they discovered $\square_{v}$, not $\square_{g}$. This nontrivial observation is indeed expected by our calculation [27].

In conclusion we have shown that the vortex lattice morphology is deeply connected to the underlying microscopic electronic structure. Specifically it is seen that the reentrant transition from the square to rhombic lattice in $\mathrm{LuNi}_{2} \mathrm{~B}_{2} \mathrm{C}$ can be well understood as arising from the two competing superconducting gap and Fermi surface anisotropies both of which are documented to exist experimentally. It is demonstrated by solving the quasiclassical Eilenberger equations numerically and analytically. We have shown another yet not found FLL transformation to the differently oriented square lattice in a higher field. In view of physical origin given, this kind of re-entrance and high field square lattice are rather generic to occur. Thus we expect a similar successive transition in type-II superconductors with four-fold symmetry at least with large $\kappa$.

We thank Y. Matsuda, N. Nishida, K. Takanaka and E. M. Forgan for valuable information.

[1] For a review see Anisotropy effects in superconductors, ed by H. Weber (Plenum, New York, 1977).

[2] D. Cribier et al., Phys. Lett. 9, 106 (1964).

[3] E. M. Forgan et al., Phys. Rev. Lett. 88, 167003 (2002).

[4] B. Obst, Phys. Stat. Sol. 45, 453 (1971).

[5] U. Yaron et al., Nature 382, 236 (1996).

[6] Y. De Wilde et al., Phys. Rev. Lett. 78, 4273 (1997).

[7] M. R. Eskildsen et al., Nature 393, 242 (1998).

[8] M. Yethiraj et al., Phys. Rev. Lett. 82, 5112 (1999).

[9] R. Gilardi et al., cond-mat/0204278.

[10] M. R. Eskildsen et al., Phys. Rev. Lett. 86, 5148 (2001).

[11] H. Sakata et al., Phys. Rev. Lett. 84, 1583 (2000).

[12] K. Ohishi et al., cond-mat/0201038.

[13] L. Ya. Vinnikov et al., Phys. Rev. B 64, 024504 and 220508(R) (2001).

[14] D. K. Christen et al., Phys. Rev. B 21, 102 (1980).

[15] K. Takanaka, Prog. Theor. Phys. 46, 1301 (1971).

[16] A. Gurevich and V. G. Kogan, Phys. Rev. Lett. 87, 177009 (2001).

[17] M. Nohara et al., J. Phys. Soc. Jpn. 66, 1888 (1997); K. Izawa et al., Phys. Rev. Lett. 86, 1327 (2001).

[18] E. Boaknin et al., Phys. Rev. Lett. 87, 237001 (2001).

[19] T. Yokoya et al., Phys. Rev. Lett. 85, 4952 (2000).

[20] Y. Matsuda, private communication.

[21] N. Nishida, private communication.

[22] See for example, S. B. Dugdale et al., Phys. Rev. Lett. 83, 4824 (1999).

[23] G. Eilenberger, Z. Physik 214, 195 (1968).

[24] M. Ichioka et al., Phys. Rev. B 59, 8902 (1999); M. Ichioka et al., J. Phys. Soc. Jpn. 66, 3928 (1999).

[25] V. G. Kogan et al., Phys. Rev. B 55, R8693 (1997).

[26] T. M. Riseman et al., Nature 396, 242 (1998).

[27] N. Nakai et al., in preparation. 